\title{
Conflacionismo epistemológico: Los estudios sobre el pentecostalismo aymara en Chile (1975-1998)
}

Epistemological conflation: Studies about Aymara pentecostalism in Chile (1975-1998)

Mg. Wilson Muñoz (wilsonsocio@gmail.com) Facultad de Educación y Humanidades, Universidad de Tarapacá (Iquique, Chile)

Dr. Miguel Mansilla (mansilla.miguel@gmail.com) Instituto de Estudios Internacionales, Universidad Arturo Prat (Iquique, Chile)

\begin{abstract}
The Aymara Pentecostalism is one of the most important religious movements in Chile. In the last decades it has been produced a considerable amount of sociological and anthropological research about this movement and this has resulted in the emergency of a particular field. However, these studies have not the thematized the relevance of the theoretical assumptions that underlie in their analysis. Due to this, our aim is to develop an epistemological analysis of the theoretical assumptions behind the studies about the Aymara Pentecostalism in Chile, elucidating specifically how they have used the difference between structure and action when they aim to understand the features of this socio-religious movement. This will allow us to elucidate the types of epistemological conflation (Archer) that take place in the explanations develop by these authors, assessing its theoretical contribution.
\end{abstract}

Key words: epistemology, structure, action, conflation, aymara pentecostalism

\section{Resumen}

El pentecostalismo aymara es uno de los movimientos religiosos más distintivos de Chile. En las últimas décadas se ha producido una considerable cantidad de estudios sociológicos y antropológicos sobre este movimiento, generándose un incipiente campo de estudios al respecto. Sin embargo, estos estudios no han tematizado la adecuación de los supuestos teóricos que subyacen a sus análisis. Por ello, nuestro objetivo es realizar un análisis epistemológico de los supuestos teóricos que subyacen a los estudios sobre pentecostalismo aymara en Chile, distinguiendo específicamente cómo han utilizado la distinción entre estructura y acción a la hora de comprender las características de este movimiento socioreligioso. Esto nos permitirá distinguir los tipos de conflacionismo epistemológico (Archer) presentes en las explicaciones esbozadas por los autores, evaluando así su rendimiento teórico.

Palabras clave: epistemología, estructura, acción, conflacionismo, pentecostalismo aymara 


\section{Introducción}

Desde las primeras investigaciones realizadas por cientistas sociales sobre el pentecostalismo chileno, hubo un claro interés por el pentecostalismo indígena en Chile (Kessler 1967, Willems 1967, D'Epinay 1968). En consonancia con el relativo éxito que tuvo este movimiento religioso dentro de la población aymara, e influido por la encendida atención que el fenómeno despertó en diversos rincones de Latinoamérica, se realizaron importantes estudios empíricos al respecto (Pérez 1975, González 1980, Guerrero 1980, González 1990, Tudela 1993, Van Kessel 2005). Inicialmente, los estudios desplegaron herramientas teóricas y metodológicas que se movieron predominantemente entre el funcionalismo y el estructural funcionalismo. Gran parte de estas investigaciones se llevaron a cabo en un contexto marcado por la profunda crisis política, social y cultural que caracterizó la dictadura militar en Chile. En este contexto, el aymara fue altamente victimizado, construcción que fue cambiando paulatinamente a medida que se usaban nuevos enfoques teóricos más cercanos al estructuralismo, como correlato de la paulatina consolidación disciplinar desarrollada en un contexto democrático.

Si bien se han generado importantes aportes al conocimiento en este ámbito, contribuyendo con ello al desarrollo de las ciencias sociales y a la antropología religiosa; esta prolífica producción no ha estado acompañada de una reflexión teórica sobre el desarrollo de los mismos estudios, como sí ha ocurrido en investigaciones sobre el protestantismo y el pentecostalismo indígena en otros contextos de América Latina. Esta situación es problemática, pues consideramos que los análisis realizados sobre el pentecostalismo aymara en Chile han tendido a recepcionar una serie de supuestos epistemológicos y teóricos de manera acrítica, los cuales luego han sido utilizados muchas veces con criterios meramente pragmáticos a la hora de analizar la información empírica. Este diagnóstico genérico se aprecia de manera significativa en el uso tácito de la distinción acción/estructura a la hora de intentar comprender y explicar diversas facetas del movimiento pentecostal aymara en Chile.

La distinción estructura/acción ha sido una de las conceptualizaciones más productivas dentro de la disciplina sociológica (Mascareño 2008). Unidad acuñada y empleada por los clásicos de la disciplina (Marx, Weber y Durkheim), fue retomada en la década de los 60 especialmente por Parsons. No obstante, a lo largo de las últimas décadas la acción ha sido puesta en el centro del debate teórico, especialmente a partir de la re-emergencia de las teorías de la acción o de la agencia, nacidas como respuesta al predominio de las teorías estructurales y estructuralistas imperantes en las ciencias sociales durante los setenta. Bajo esta etiqueta se pueden incluir a una pléyade de propuestas, como la teoría de la estructuración (Giddens), la teoría de la acción comunicativa (Habermas), la teoría de los campos y habitus (Bourdieu), la teoría morfogenética (Archer) o la teoría de la acción creativa (Joas), por nombrar algunas de las propuestas más significativas de la sociología contemporánea.

Si bien todos los autores recién mencionados han establecido algún nivel de reflexión respecto a la distinción acción/estructura utilizada en las ciencias sociales, evaluando y defendiendo la plausibilidad teórica de sus propias propuestas; ha sido Margaret Archer quien ha desarrollado una reflexión metateórica destinada específicamente a analizar los supuestos ontológicos y epistemológicos presentes en las teorías socioculturales, distinguiendo de manera especial el uso de la distinción entre estructura y acción. En esta línea, la obra de Archer ha recibido una reciente atención por parte de autores que han apreciado en su propuesta una conceptualización clara y útil para realizar análisis teóricos en diversos ámbitos de la teoría social. Nosotros nos sumaremos a esta línea y utilizaremos específicamente la propuesta de Archer (1997), pues consideramos que nos permitirá analizar de mejor manera cómo las 
diversas investigaciones sobre el pentecostalismo aymara han utilizado la distinción entre acción y estructura en su análisis.

Archer se ha centrado especialmente en el fenómeno de la morfogénesis sociocultural, sosteniendo que los procesos producidos por intercambios sociales complejos no solo producen cambios en la estructura del sistema, sino también conducen a la elaboración estructural de la sociedad. En esta línea, la socióloga argumenta que existen propiedades emergentes que producen esta transformación, tanto en el plano de las acciones como de las interacciones que las produjeron. Así, una vez que las estructuras han emergido, pueden reaccionar frente a la acción y la interacción, alterándolas a su vez.

Bajo la propuesta teórica morfogenética, la autora ha analizado este fenómeno en el tiempo, considerando la existencia de un sinnúmero de ciclos y secuencias de transformaciones estructurales, donde se producen alteraciones en los planos de la acción, las estructuras y su interacción para la elaboración estructural. De ahí el interés de la autora por distinguir analíticamente los planos de la acción y la estructura en las explicaciones socioculturales.

Es a partir de esta línea interpretativa que Archer ha desarrollado una propuesta conceptual específica para comprender las estructuras ontológica y epistemológica utilizadas por las teorías socioculturales para explicar el origen y desarrollo de lo social. A grandes rasgos, sostiene que según la definición y uso de los supuestos epistemológicos presentes en las teorías socioculturales, sus explicaciones teóricas pueden ser catalogadas bajo tres grandes categorías. Por un lado, señala que existe un (a) conflacionismo ascendente cuando las teorías presuponen la determinación de las estructuras por la acción. En estas propuestas si bien se reconoce la autonomía de la acción, se elude la autonomía de las estructuras (por ejemplo, como ocurre en las teorías de la rational choice). Por otro lado, existe un (b) conflacionismo descendente cuando las teorías presuponen la determinación de la acción por la estructura, pero no reconocen la autonomía de la acción (por ejemplo, el sistema social concebido por Parsons). En términos más exactos, Archer entiende el conflacionismo ascendente y descendente de la siguiente forma: "En un caso las propiedades culturales simplemente son formadas y transformadas por algún grupo dominante libre de trabas o puestas a merced de caprichosas renegociaciones por una agencia irrestricta. En el otro, algún código cultural o sistema central de valores impone su coreografía a la vida cultural y los agentes se reducen a Träger o portadores de sus propiedades, ya sea a través de la socialización excesiva o de la mistificación" (Archer 1997:13).

Finalmente, Archer sostiene que existiría un tercer caso denominado (c) conflacionismo central, el cual ocurriría cuando las teorías reconocen la existencia de acción y estructura en la explicación sociocultural, pero fusionan o integran ambas instancias por medio de un tercer término (por ejemplo, bajo el concepto de prácticas en Giddens o habitus en Bourdieu. De manera que el conflacionismo central se caracterizaría porque "la elisión se produce en el 'medio'. Lo que pasa en este caso es que se niega autonomía tanto a las 'partes' como a la 'gente', cosa que tiene precisamente el mismo efecto de excluir todo examen de su juego recíproco" (Archer 1997:13), pues las propiedades se entremezclan, haciéndose indistintas.

Una importante sentencia que se desprende de esta clasificación es que, según sostiene la autora, cualquier tipo de conflacionismo no logra dar cuenta efectivamente de la autonomía de la acción y la estructura, ni de los órdenes emergentes que puedan generar, de ahí que no estén en óptimas condiciones de comprender y captar las complejidades de lo social. Este diagnóstico esgrimido por 
Archer se puede apreciar claramente en gran parte de los estudios sobre el pentecostalismo aymara en Chile, especialmente a través de la figura del conflacionismo descendente, como intentaremos mostrar en nuestro análisis. Por ello, consideramos que la distinción analítica sobre el conflacionismo teórico es bastante útil para analizar cómo las teorías utilizadas en nuestro caso de estudio han tratado la distinción entre estructura y agencia en sus explicaciones.

En concreto, uno de los aspectos que permite situar y ordenar estas teorías sobre el pentecostalismo aymara es distinguir el peso relativo y la autonomía que cada una de las propuestas le otorgan en su explicación a la estructura, la acción y la naturaleza del vínculo relacional que las une. Esto permitiría apreciar las diferencias que existen entre los supuestos ontológicos y epistemológicos que cada investigación posee. Por ejemplo: ¿Cómo se concibe la estructura cultural? ¿Cómo son concebidos los planos de la acción y la agencia en las explicaciones? ¿Cuál es la concepción del aymara y del pentecostal como actores sociales? Todos ellos cuestionamientos absolutamente relevantes para las investigaciones analizadas en nuestro caso de estudio, así como también para muchas de las investigaciones y reflexiones antropológicas y sociológicas generadas sobre la religiosidad popular en general.

El problema con el que nos encontramos es que debido a la inexistencia de análisis teóricos sobre el pentecostalismo aymara en particular, este nutrido debate epistemológico ha estado ausente. La ausencia de investigaciones metateóricas ha conllevado a la reproducción acrítica de la distinción acción/estructura, dejando intocada la problematización de los supuestos ontológicos y epistemológicos sobre los cuales se yerguen los hallazgos empíricos de estas investigaciones. Sobre esta base se ha construido una imagen del pentecostalismo aymara y del pentecostal aymara que lo describe como un actor pasivo, víctima y con bajísima capacidad de reflexión, resistencia o de producción de símbolos y contenidos culturales. Si bien gran parte de estas investigaciones sobre el pentecostalismo aymara han sido reales aportes al conocimiento, esta desatención epistemológica se presenta como una de las limitantes actuales más importantes para el futuro desarrollo de este ámbito específico, así como también para el desarrollo de la sociología y de la antropología de la religión en la región.

Por ello consideramos que el desarrollo de trabajos metateóricos como éste son de vital importancia, pues el análisis propuesto no solo permitirá distinguir más claramente cuáles han sido los supuestos teóricos y epistemológicos que han fundamentado a estos estudios, sino que además ofrecerá lineamientos críticos que podrían contribuir directamente al desarrollo de nuevos enfoques y propuestas teóricas para iluminar el fenómeno del pentecostalismo aymara.

\section{Los estudios sobre el pentecostalismo aymara en Chile}

El pentecostalismo arribó al altiplano andino el año 1957 de la mano de Braulio Mamani, quien se convirtió al pentecostalismo mientras realizaba su servicio militar en la pequeña ciudad de Diego de Almagro (ex Pueblo Hundido). Mamani realizó su conversión al alero de la Iglesia Evangélica Pentecostal, reconocida como la iglesia más conservadora y tradicional del pentecostalismo chileno. Don Braulio se convirtió prontamente en uno de los misioneros protagonistas de la conversión aymara al pentecostalismo, evangelizando el altiplano chileno y posteriormente el boliviano, luego de su traslado a Sibaya (Bolivia) en el año 1964. En ambos lugares, las conversiones fueron sistemáticas y sostenidas en el tiempo, básicamente porque las personas que se convertían lo hacían normalmente acompañadas de todo su grupo familiar. 
En el altiplano chileno el pentecostalismo aymara fue tempranamente estudiado. Sin embargo, los investigadores desconocían los trabajos sobre el pentecostalismo chileno antes realizados, especialmente el conocido y significativo libro de D’Epinay (1968). Desde un inicio este movimiento religioso fue investigado como una secta, de manera que a los conversos aymaras no se les reconocía primeramente como indígenas, sino como sectarios, concepción que cambió a comienzos de la década de 1990 (González 1990, Tudela 1993, Guerrero 1994). En concreto, se partió concibiendo al pentecostalismo como una secta protestante que exigía a los aymaras una renuncia total de las costumbres tradicionales. Sin embargo, el desarrollo de estas investigaciones sentó un precedente importante en el ámbito de las ciencias sociales, siendo pioneros en el estudio de este fenómeno religioso en la población aymara.

En general, estas investigaciones muestran la relevancia empírica de la acción social, tanto de los conversos pentecostales como de quienes desarrollaron una resistencia cultural (aymaras); lo que evidencia en cierta forma la importancia que adquirió también en términos analíticos el plano de las acciones en el desarrollo del pentecostalismo aymara. Sin embargo, la explicación que ciertamente predominó fue una lectura estructural a la hora de comprender el origen y desarrollo de las acciones de los aymaras y de los pentecostales, manifestando un determinismo que puede ser leído como una forma de conflacionismo descendente.

Para evidenciar esta tendencia analítica, a continuación pasaremos revista a los principales estudios e investigaciones realizadas al respecto, prestando especial atención al tratamiento teórico y epistemológico que los autores han realizado de los conceptos de estructura y acción (agencia). De esta forma podremos evidenciar la presencia y especificidad de los tipos de conflacionismo epistemológico presentes en esta literatura, los cuales serán evaluados en las conclusiones finales.

\section{El dominio de la estructura}

La tradición de estudios sobre el pentecostalismo aymara en el norte de Chile, sobre todo durante sus inicios, estaba especialmente seducida por la idea del implacable peso que las estructuras socioculturales poseían sobre los actores. Especialmente cuando se concibió al pentecostalismo como un movimiento social totalmente ajeno y extraño a la zona andina, que poco a poco iba imponiendo una lógica foránea en tierras aymaras. Recién iniciada la década de los '80, Guerrero aseguraba que el movimiento pentecostal se presentó ante un individuo aymara desarraigado de su tradición cultural, a quien se le ofrecía e imponía un modo específico de "definir y percibir la realidad, sus relaciones con la sociedad circundante, sus sistemas de valores y todo un cuerpo de creencias" (Guerrero 1980:10). Fácilmente podríamos sostener que hubo una preocupación por el pentecostalismo, pero no por los pentecostales; hubo una preocupación por la estructuración que el pentecostalismo imponía a las acciones de los conversos aymaras, pero no por el efecto que tenían las acciones de los conversos aymaras sobre las estructuras culturales.

Para los investigadores, la situación de anomia, desarraigo y soledad social que afectaba a la población aymara generó una tierra fértil para la producción de una gran población de campesinos alienados de la cultura (aymara): una presa fácil para el pentecostalismo (Pérez 1975, González 1980, Guerrero 1980). Debemos concederle a los autores que la figura de la anomia y el desarraigo expresaba una situación histórica que vivía la población aymara (Gundermann y González 2009a, Gundermann y González 2009b). Sin embargo, la construcción del sujeto aymara realizada por los investigadores de este 
fenómeno socioreligioso, especialmente durante la década de los 80 , aparece totalmente desprovista de cualquier forma de resistencia cultural, básicamente porque, en el fondo, se afirmaba que este grupo social no poseía afincada cultura alguna. En este sentido, el movimiento pentecostal aparece invadiendo culturalmente a la población, imponiendo una cultura dominante, aplastante y efectiva en su accionar.

Guerrero utiliza la distinción marxista entre estructura y superestructura para entender el tipo de dominación cultural a la que se vio sometida la población aymara por parte del movimiento pentecostal. En concreto, el sociólogo contempla a los aymaras como un grupo sometido bajo el modo de producción imperante a nivel nacional, donde juega un papel totalmente subordinado en el contexto del desarrollo de un orden capitalista emergente. En esta línea, el movimiento pentecostal se alza como un evidente agente desestructurador, un claro abanderado de los intereses nacionales. De esta forma, haría "extensiva la desestructuración a nivel de la superestructura", pues este movimiento religioso "va paulatinamente legitimando un orden socio-económico marcado por la dependencia y por las relaciones desiguales que la sociedad andina mantiene con la sociedad nacional" (Guerrero 1980:7). Por lo tanto, aunque existe acción, ésta prontamente se ve constreñida y aplastada por las estructuras. Es decir, en el plano analítico la acción es concebida como un epifenómeno del orden estructural, lo que puede ser entendido fácilmente como la utilización de un conflacionismo descendente en las explicaciones de los autores, en tanto conciben al orden social como estructurado y generado primordialmente en este nivel operativo.

Sin embargo, la relación de determinación entre estructura y acción (actores) no aparece siempre del todo transparente en el plano epistemológico. Ante la situación de crisis cultural vivida por los aymaras y la intromisión del movimiento pentecostal, los investigadores asumen que la población indígena manifiesta un nivel de agencia en sus decisiones. Al menos en el plano del sentido. Así, Guerrero señala que el hombre aymara asume la conciencia de que puede vivir desapegado de su religión autóctona. Sin embargo, este nivel de agencia expresada en la conciencia de los sujetos, resorte intencional último de sus prácticas y acciones, está determinado por la imposición de una cultura dominante foránea que rechaza a la tradición indígena. $Y$ es así como "se empieza a re-socializar en un extraño sistema de valores que le otorga el movimiento pentecostal al extremo de renegar de su cultura y tradición" (Guerrero 1980:30). De manera que el tratamiento teórico que tanto Guerrero como otros autores realizan de los sujetos y el sentido de su acción, es un tanto paradójico, pues se trataría de una especie de actor que es consciente de decidir su inconsciencia. En este sentido, no solo se elude realmente la supuesta capacidad de agencia del actor, sino que además, cuando se manifiesta, aparece predominantemente descrita como una estrategia con ribetes masculinos, generada casi únicamente por personas adultas y que, en alguna medida, poseen algún estatus de prestigio o poder.

Esta sobre-determinación de parte de la estructura sociocultural pentecostal evidencia el poder que se le otorga al plano macrosocial en la configuración del orden de la sociedad. Entendido como un movimiento sociorreligioso que danza al ritmo de los valores dominantes de la sociedad nacional, tanto Guerrero como González sostienen que, en el plano meso-social, el pentecostalismo opera de manera bastante similar a la institución de la escuela, especialmente en lo que se refiere al poder destructivo que posee al atacar las organizaciones sociales distintivas de la cultura aymara. En este sentido, ambos autores destacan el poder que poseen las estructuras, no sólo en el plano individual de la acción social, sino también sobre instituciones como la familia, reproduciendo una vez más la figura del conflacionismo descendente, pero en un nivel sociológico intermedio referido al plano de las organizaciones o instituciones sociales. No obstante, entre ambos autores se aprecia una leve diferencia respecto a la 
intensidad del peso que le otorgan a las estructuras en sus explicaciones, pues pese al determinismo estructural sostenido explícita e implícitamente por Guerrero en sus explicaciones, al final del día el autor parece dejar la ventana abierta al desarrollo de cierto nivel de agencia pentecostal aymara; mientras que la visión de González parece dejar menos margen a esta posibilidad, como se aprecia en el siguiente párrafo: "la escuela como el pentecostalismo desintegran a la estructura familiar aymara, acercándola a los valores y pautas de conducta urbana-moderna y, acentuando por esa vía, la dominación socio-cultural de la unidad familiar con respecto a la sociedad nacional y su sistema urbano" (González 1980:34).

González nos advierte que si bien la familia pentecostal considera el modelo del ayllu como punto de partida, esto no se puede considerar como una manifestación de la continuidad cultural, pues se trataría más bien de una pseudo revitalización de la familia aymara tradicional. Argumenta que el modelo familiar aymara es totalmente redefinido por la cultura pentecostal, sacado de su contexto original e inserto en una cultural extraña, haciéndola formar parte de una "doctrina alógena sustentadora de ideas, pautas de conducta y valores que contradicen a los de la estructura familiar autóctona" (González 1980:35). En el fondo, la desintegración de la estructura familiar aymara se produce por el acercamiento que realiza el pentecostalismo a los valores y pautas de conducta propias de la sociedad urbana moderna. Es a través de esta vía que este movimiento encarna y reproduce una larga historia de dominación socio-cultural de la organización familiar, emanada y apoyada especialmente por la sociedad nacional y su sistema urbano. En esta lógica, son las estructuras socioculturales dominantes propias de la sociedad nacional las que determinan el éxito del pentecostalismo andino, por la vía de la destrucción de la cultura aymara. Bajo esta explicación conflacionista descendente, las acciones continúan siendo determinadas estructuralmente, de manera que los niveles de agenciamiento de los actores se ven determinados externamente, destinados finalmente a no influir mayormente en la generación de este nuevo orden sociocultural que se está fraguando.

\section{Oposición y contradicción}

En la argumentación de la mayoría de los autores la determinación sociocultural de los actores sociales aparece provocada por el poder que posee la estructura cultural sobre ellos. Sin embargo, la intensidad del poder de destrucción aparece enfatizada por la profunda discontinuidad cultural que existiría entre la tradición aymara y la doctrina pentecostal. Guerrero argumenta que desde que el movimiento pentecostal arribó con el primer aymara converso a la zona andina, se inauguró un proceso profundo y duradero de una serie de incompatibilidades, alcanzando incluso manifestaciones violentas que perdurarían hasta la actualidad. De ahí que la relación entre la comunidad aymara y pentecostal se caracterice por "la existencia de un tenso antagonismo" (Guerrero 1980:30) que finalmente evidencia una profunda incompatibilidad estructural.

De hecho, para el aymara tradicional el mundo es arquetípicamente bueno, fuente de toda bondad y generosidad, de manera que tanto la estructura cultural como las acciones adquieren valoraciones morales. Sin embargo, para el pentecostal aymara el mundo es la génesis de toda maldad, símbolo de idolatría e incredulidad, pues se trata de un mundo eminentemente pecaminoso. Por ello las actividades mundanas practicadas tradicionalmente por los aymaras, como las fiestas, bailes, el consumo de coca, alcohol y cigarrillos, aunque también la acción política y social, entre muchas otras; son malas y prohibidas, o en el mejor de los casos, pueden ser levemente toleradas. Sin embargo, pese a que el pentecostalismo concibe las actividades aymaras como actividades profanas, ellos mismos crean y 
recrean actividades llenas de sentido, con un marcado carácter ritual y religioso aymara. Por lo tanto, es cuestionable la idea de que se trate exactamente de una ruptura cultural, pues la figura parece obedecer más a un proceso de redefinición cultural. Tal como sostienen los mismos autores, gracias a los mismos rituales los aymaras establecen diversas formas de comunicación con lo divino. De hecho: "Hasta las actividades económicas, como la siembra, la construcción de una casa, un viaje, etc., se inscriben en un contexto religioso y se acompañan de un ritual productivo" (Van Kessel y Guerrero 1987:35).

Aquí nos encontramos con una paradoja analítica, pues pese al peso estructural que los autores le otorgan a la cultura aymara concebida dentro de la población indígena, de todas formas dejaban cierto espacio de libertad a la acción social en sus explicaciones. Sin embargo, bajo las nuevas estructuras culturales que impone el pentecostalismo aymara, los autores tienden a sostener que esta libertad de acción se pierde. Pero las estructuras de socialización cultural aymara de los conversos no pueden desaparecer por el puro acto imperativo de las normas pentecostales. Esto lleva a pensar que en algún sentido las nuevas acciones ofrecidas por los pentecostales a la población aymara estaban marcadas e influidas por sus estructuras culturales tradicionales, de lo contrario el tránsito no podría explicarse completamente en este caso.

En el plano ideológico, Guerrero argumenta constantemente que el pentecostalismo se alinea perfectamente con los ideales y valores de la modernidad, lo que se manifiesta en que ambos sostienen la existencia de una "oposición radical de lo sagrado y lo profano" (Guerrero 1984:5). Sin embargo, la ideología pentecostal realizaría una especificación de este argumento, entendiendo a todo lo aymara y tradicional como perteneciente al mundo profano; de manera que la adhesión ideológica y práctica a esta tradición simplemente produciría la perdición de los sujetos y el eterno subdesarrollo de su sociedad. Por ello el llamado es a convertir este mundo profano en sagrado, es decir, moderno y pentecostal, aunque sea a costa de su total destrucción.

Uno de los ámbitos sociales donde se manifiesta de manera clara esta relación de ruptura estructural en el plano cultural entre la cosmovisión aymara y pentecostal es en la medicina o la curación. No obstante, y a diferencia de la argumentación anterior, es importante mencionar que en este análisis Guerrero y Van Kessel señalan la existencia simultánea de procesos de continuidad y de ruptura entre ambas ideologías. Por lo tanto, pareciera que las nuevas acciones contribuyen a gatillar ciertas redefiniciones estructurales. Llama especialmente la atención esta nueva interpretación basada en el determinismo estructural de la cultura, pues si bien inicialmente se le otorga cierto margen de agencia a los sujetos aymaras, luego se le es arrebatada en la mayor parte de la interpretación que intenta comprender la interacción existente entre la cosmovisión aymara y la doctrina pentecostal, evidenciando nuevamente con ello el predominio de un conflacionismo descendente en sus explicaciones. Señalan los autores: "EI movimiento pentecostal pretende provocar un vuelco radical en la cosmovisión de los andinos y establecer un nuevo orden o Cosmos. Este vuelco radical o 'Kuti' es un concepto básico en la filosofía andina y expresa el vuelco, o fin, de un 'Pacha' (Mundo o era) para dar origen a otro cosmos. En la mitología andina existen varias épocas sucesivas que preceden a la actual. Esta misma conciencia, la expresa la visión historiográfica de los Incas referente a su propio pasado. A partir de este concepto y conciencia de 'Kuti'; siempre vigente en el mundo andino, podemos apreciar los elementos de continuidad y de ruptura en el sistema de salud, en el saneamiento y en la terapia administrada por los pastores pentecostales, los que pretenden desplazar a los Yatiris andinos" (Van Kessel y Guerrero 1987:36). 
Respecto a los rituales de sanación utilizados por la medicina aymara y pentecostal, los sociólogos señalan que existe una clara relación de continuidad, pues en ambas cosmovisiones concurren una serie de elementos extra-físicos en el origen y curación de la enfermedad; en concreto, se manifiestan una serie de elementos andinos míticos como también explicaciones religiosas derivadas de la interpretación de la Biblia. Así se evidencia la existencia de cierta homología estructural en el plano cultural, la cual se manifestaría también en el ámbito del sentido atribuido por los actores sociales a sus acciones. Sin embargo, aquí se acaba el plano de las semejanzas, pues luego ambos autores continúan sosteniendo la tesis de la oposición cultural en el nivel estructural, alimentando con ello nuevamente un conflacionismo descendente. En términos más específicos, argumentan que los actores sociales que participan en estos rituales "lo hacen motivados por horizontes culturales e ideológicos totalmente distintos" (Van Kessel y Guerrero 1987:14). De manera que en el plano de los actores sociales, tanto el Yatiri como el pastor pentecostal, actores principales de cada una de estas organizaciones, están referidos finalmente a tradiciones diferentes y contradictorias entre sí, pese a que ambos forman parte de la tradición andina contemporánea.

Las oposiciones y contradicciones culturales pueden observarse clara y profundamente en el plano de la operación ritual y la cosmovisión que acompaña a cada una de las prácticas que realizan ambos grupos sociales. Ambos autores argumentan que uno de los aspectos que estructura esta diferencia es la cosmovisión, pues articula la esfera cultural de cada uno de estos colectivos. La cosmovisión andina concibe la existencia de una divinidad inmanente, lo cual permite al aymara la plena acepción del mundo, entendido este último como un medio natural y lleno de sentido con el cual se identifica y en el cual se proyecta. Por su parte, la cosmovisión pentecostal concibe la existencia de una divinidad trascendente, obligando con ello al creyente a rechazar el mundo y todas las actividades mundanas desarrolladas en él, pues carecen de sentido y son pecaminosas. Esta oposición ideológica en el plano cultural, determina y desemboca directamente en el plano de la praxis de los actores, en específico en el plano ético, evidenciando de manera clara cómo se estructura la explicación conflacionista del orden sociocultural, donde la estructura cultural aparece como un manto que suele cubrir todas las estrategias y acciones individuales. De hecho, la metáfora utilizada por los autores evidencia la profundidad de esta contradicción y su determinación, pues ambos sostienen que "las consecuencias de las dos cosmovisiones en el plano ético, son tan fuertes que allí se contraponen como una foto a su negativo" (Van Kessel y Guerrero 1987:35).

De manera que la idea de la contradicción y oposición cultural es claramente predominante en estos autores. Pareciera entonces que de alguna u otra forma, el aymara pentecostal sintetiza y cristaliza esta profunda discontinuidad ideológica que existe entre el movimiento pentecostal y la sociedad aymara. Este sincretismo simplemente reflejaría "el colapso casi total de la consciencia social aymara" (Guerrero 1980:4), manifestando con ello la clara sobre-determinación estructural de la cultura pentecostal sobre la sociedad y los actores andinos. Pero la sobre-determinación referida por Guerrero posee una profundidad de más de cuatro siglos, donde el pentecostalismo es concebido simplemente como una consecuencia histórica de la dominación, sub-desarrollo y anomia que ha acompañado a esta sociedad. Quizás sea esta idea la que expresa de manera más extrema la figura epistemológica del conflacionismo descendente, donde las acciones y estrategias de los actores aparecen pre y sobre determinadas desde el inicio de los tiempos por las estructuras de la dominación cultural. 


\section{La (limitada) agencia}

Hemos podido ver claramente cómo el fenómeno de la agencia, entendida como la capacidad (potencial o real) de los actores de influir en la determinación de sus acciones, parece fuertemente determinada por las estructuras culturales en nuestro caso de estudio. No obstante, igualmente hay espacios donde parece deslizarse una cierta autonomía en el plano de las acciones, no tanto en el nivel de los sujetos individuales, sino más bien de los sujetos entendidos colectivamente, dejando ver cierto nivel de agencia sociocultural, aunque ciertamente limitada.

Como señalábamos, para investigadores como Pérez, González y Guerrero la presencia del movimiento pentecostal se convirtió en un problema intolerable, pues ponía en jaque a toda la estructura social aymara. Esta figura se aprecia en el plano ideológico, donde se asegura que el pentecostalismo ha contribuido a la agudización del proceso de sub-desarrollo, donde su secuela más trágica ha sido la "debilitada conciencia social aymara" (Guerrero 1980:38). Guerrero continúa argumentando que esto es lo que ha permitido que el pentecostalismo pueda ser visto como "una alternativa religiosa más por parte de los aymaras" (Guerrero 1980:38). Esta lectura, si bien epistemológicamente sigue operando bajo el mecanismo de la determinación estructural, evidencia cierto nivel de agencia en el plano de la acción y la conciencia de los actores sociales. Por un lado, el autor muestra que la intrusión del pentecostalismo ha agravado el declive cultural de la población aymara, manifiesto en su alicaída conciencia cultural. Pero pese a este debilitamiento que observa en el plano de la conciencia de los actores, el autor de todas formas le asigna un poder o grado de agencia a este plano operativo, pues son los actores aymaras conversos quienes en última instancia advierten y conciben al pentecostalismo como una alternativa religiosa más. Es decir, son ellos quienes construyen al pentecostalismo como una competencia válida frente al catolicismo popular aymara (1). En ello destaca: "Esta oferta del movimiento pentecostal es altamente atractiva, sobre todo si se presenta en un contexto de pérdida o de deslegitimación de la identidad cultural andina. Los que ingresan al pentecostalismo, lo hacen en busca de una identidad cultural perdida. El movimiento pentecostal ofrece eso y lo hace a través de la solución de problemas concretos, en este caso, en la búsqueda de la salud" (Guerrero 1992:34).

La metáfora del mercado religioso, ampliamente utilizada por la sociología y la antropología para describir contextos de diversidad religiosa contemporánea, evidencia la concepción de la religión como parte de un mercado donde se generan competencias entre distintas ofertas religiosas y donde los potenciales demandantes pueden, en la medida de lo posible, escoger la alternativa que les sea más atractiva. Al igual que la economía moderna que basa la predicción de las operaciones microeconómicas en el cálculo que puedan realizar los actores sociales comportándose "como si" fueran racionales, maximizando así la utilidad de su elección, de igual manera concibe Guerrero a los sujetos aymaras. Es decir, sin desconocer el determinismo estructural aplastante que significa la introducción del pentecostalismo, los actores y futuros conversos pueden escoger su pertenencia religiosa en este nuevo escenario sociocultural concebido como mercado religioso.

Evidentemente esta decisión no es concebida de manera netamente individual. Aunque las decisiones de los conversos deban ser entendidas como decisiones agenciales, Guerrero concibe la actuación del pentecostalismo como un mecanismo funcional a través del cual la modernidad y el capitalismo se introduce crecientemente en los Andes. Es decir, nos encontramos con una explicación estructural funcionalista que permite convertir a estas manifestaciones religiosas étnicas en funcionales para el sistema. De hecho, el autor argumenta que la ideología pentecostal posee una función bastante 
delimitada: otorgar a la modernidad que acecha al altiplano cierto estado de coherencia y unidad. Por lo tanto, pese a que le otorga sentido y poder al plano de la acción, esta adquiere ribetes negativos, donde, una vez más, el pentecostalismo es la expresión en el plano religioso de un capitalismo claramente dependiente. Por ello es en el ámbito discursivo donde se "entrega y da a la vez una dimensión y justificación religiosa a una multitud de elementos que aparecen como típicos y exclusivos del capitalismo, la competencia, el individualismo, el consumismo, etc." (Guerrero 1984:5). De esta forma, se advierte la presencia de un determinado nivel de agencia, aunque determinado estructuralmente a nivel social y cultural.

Uno de los fenómenos donde se aprecia claramente la dimensión agencial es en la conversión religiosa, una de las manifestaciones más distintivas del pentecostalismo. Guerrero entiende que si bien la estructura cultural es la base sobre la cual se tejen todas las articulaciones de actores y organizaciones sociales, comprende también que la conversión, "con todos sus modos de objetivarse, física y verbalmente sólo es posible en la medida en que el futuro converso vea en la nueva oferta religiosa un mundo relativamente coherente y conocido. De otro modo no se podría entender la conversión de un católico al mundo pentecostal" (Guerrero 1998:119). La lectura weberiana utilizada por el sociólogo, deposita en el plano del sentido -subjetivamente mentado por los actores, pero culturalmente estructurado por la sociedad- la respuesta última que permite entender este fenómeno, altamente significativo para el movimiento pentecostal en la región. Sigue primando, no obstante, ese privilegio explicativo otorgado al mecanismo de la homología estructural en el plano cultural, donde solo aquello que parece coherente al sentido tradicional puede ser recepcionado efectivamente.

En términos más concretos, podríamos señalar también que se trata de una especie de agencialidad abstracta, pues si bien le otorga una aparente poder de agencia a su explicación a través del fenómeno de la conversión, no le asigna importancia alguna al converso. Básicamente porque concibe a la conversión más como un resultado esencialmente rupturista que como un proceso relacional. Es decir, no solo no se entiende finalmente cómo resulta la relación entre estructura y acción, sino que tampoco se explicita la relación entre la acción y el actor. Además, el único agente relevante para los investigadores pareciera ser el pastor, de manera que la agencia del converso se ve obnubilada por la presencia de este líder carismático, como veremos más adelante. De manera que se aprecia una especie de conflacionismo ascendente, donde la explicación de un fenómeno social descansa en el plano de la acción, aunque no se explique concretamente el rol del converso en sí mismo.

Podemos encontrar también un resquicio de aquello que la teoría ampliamente denomina como la agencia no solo en los actores aymaras, sino también en los aymaras pentecostales: "Lo anterior [la conversión] se relaciona con la capacidad que tienen los aymaras para leer lo que está sucediendo en su sociedad; lectura subjetiva, marcada por su propia biografía y por sus propios ritmos emocionales, afectivos y religiosos y por el tipo de relaciones interpersonales que construya. Se trata, en suma, de ver cómo el aymara, en un momento determinado, supera la tensión entre lo que ocurre a nivel macro con lo que le está ocurriendo en términos personales. La conversión es entonces, desde este punto de vista, una solución a la crisis que le acontece al individuo. En otras palabras, es una solución de sentido" (Guerrero 1998:111).

Es importante apreciar aquí la presencia de una especie de indistinción epistemológica. Mientras que por un lado aparece una vez más aquella determinación cultural externa y dominante sobre los aymaras, por otro lado aparece un actor que tiene la capacidad de realizar una lectura agencial, subjetiva e histórica 
de la situación de crisis que está atravesando a nivel personal. Como señala Guerrero, la conversión es finalmente el mecanismo funcional que permite, tanto subjetiva como estructuralmente, dar respuesta a esta compleja situación que enrola al sistema social y los actores. Sin embargo, esta paradoja conceptual encierra ciertas dificultades que se manifiestan epistemológicamente en una especie de conflacionismo central, pues en este punto es difícil realmente determinar dónde se ubica el principio explicativo de un problema tan complejo como el de la conversión (¿estructura?, ¿acción?, ¿ estructura-acción? o ¿acciónestructura?). La solución intermedia propuesta no distingue entre el funcionamiento de la estructura y la agencia, rompiendo con el esquema explicativo propuesto desde un inicio por los autores para explicar este fenómeno religioso, además de sembrar fundadas dudas sobre cómo se concibe la operación real del encuentro existente entre el movimiento pentecostal y la sociedad aymara en el norte de Chile. Este último es el problema más importante que ha acompañado a toda la literatura sobre el pentecostalismo aymara en la región (latinoamericana), tanto en el plano sociológico como antropológico.

Finalmente, Guerrero vuelve a dotar de poder agencial a los actores sociales, señalando que quienes en virtud de la conversión al pentecostalismo han alcanzado un nuevo estatus social, tienden a priorizar su denominación pentecostal por sobre su denominación tradicional aymara. Cifras en mano, el sociólogo señala que en la década de los ' 90 fueron estos últimos quienes alcanzaron un crecimiento espectacular en la Comuna de Colchane (altiplano andino). Según el autor, en este contexto tanto la presencia activa del pastor en Cariquima, como el cargo de alcalde ostentado por un evangélico, permitieron configurar una situación de duplicación e intensificación mutua de estatus y poder, lo cual impactó directamente en la influencia que podían tener en la zona, explicando parcialmente así el "crecimiento que podríamos llamar de espectacular" (Guerrero 1993:36) del movimiento pentecostal en este lugar.

\section{El Pastor: un súper agente}

Pero donde se manifiesta de manera más clara el poder de la agencia pentecostal, incluso de manera algo exagerada, es en la construcción conceptual (ideal) que realizan todos los autores del pastor pentecostal. En este sentido, la literatura no solo ha caído en un claro pastorcentrismo a la hora de comprender el fenómeno del éxito y desarrollo del pentecostalismo aymara en el norte de Chile, sino que esta predilección ideal se expresa también en el plano de la elaboración de los conceptos teóricos que permiten describir mecanismos sociales que articulan lo social, donde la figura del pastor aparece como una especie de "súper actor" o "súper agente"; contraviniendo en gran medida la lógica explicativa propuesta para describir y explicar el éxito de este movimiento socioreligioso. Siguiendo a Archer, podríamos señalar que esto manifiesta de manera clara la presencia de un conflacionismo ascendente en las explicaciones de los autores, donde la generación y desarrollo del pentecostalismo aymara aparece originado y determinado en gran medida por la agencia de estos actores claves.

González llega a decir incluso que "el rol clave en la eficacia del pentecostalismo lo constituye el Pastor, actor que se desempeña como 'padre protector' de la neo-familia pentecostal en virtud de sus cualidades y méritos personales" (González 1980:36). En este sentido, la figura del pastor opera culturalmente como un potente agente de resocialización, especialmente en el círculo de los actores aymaras conversos. Debemos recordar que prácticas de abstinencia como no beber y no fumar, unida a la idea de trabajar para "progresar" y tener éxito, son centrales dentro de la cosmovisión de la familia pentecostal y se ven constantemente enfrentados a las costumbres, ritos, fiestas y ceremonias autóctonas andinas. Al igual que Guerrero y Van Kessel, González observa una clara "contraposición valórica que se resuelve a favor del pentecostalismo" (González 1980:36), pues finalmente es este 
movimiento el que ordena a sus adeptos apartarse del mundo pagano aymara, en tanto este último no permite civilizarse ni tener éxito ni progreso material. Sin embargo, si este juego de poder manifiesto en la dimensión simbólica y cultural finalmente arroja como vencedor al pentecostalismo, es porque la figura del pastor posee una altísima eficacia simbólica y ritual dentro de la población conversa, lo que permitiría mantener la adhesión de gran parte de los conversos y explicaría la rapidez de este proceso de transformación identitaria.

Una de las limitaciones clave en estos análisis es que el único actor-agente que aparece en estas investigaciones es el pastor, mientras que los laicos son sujetos-actores que sólo reproducen, recitan y obedecen libretos previamente delineados por este líder carismático. El pastor es el único agente que tiene voz y capacidad de decisión, además de ser el interlocutor válido y tener el poder de decidir por la comunidad. Pareciera entonces que el pastor cumpliera la función que inicialmente le otorgaban los mismos autores a la estructura cultural, pues aparece como un agente todo poderoso y divinizado. Sin embargo, Sergio González muestra que en el plano simbólico, aunque el pastor sea una autoridad, "aún prevalece la función ganadera-aymara por sobre la función religiosa-pentecostal" (González 1990:43), lo que significa que su autoridad descansa principalmente en un fundamento más social que familiar, matizando así la tesis de los autores.

Otra manifestación del poder agencial adjudicado al pastor es descrito por Guerrero, quien sostiene que el pastor pentecostal evangeliza las tierras paganas de los Andes y el mejor camino para lograrlo es "modernizando el altiplano, lo que equivale a destruirlo como sociedad y cultura" (Guerrero 1984:8). Agrega más adelante que solo cuando la sociedad andina desaparezca totalmente como tal, la venida del Señor será cierta. Para lograr este cometido, el pentecostalismo deberá preparar el camino y seguir cumpliendo su principal función sociopolítica: "aniquilar estructuralmente la sociedad [aymara] y construir sobre sus ruinas una sociedad moderna" (Guerrero 1984:11). Así, aunque el pentecostalismo es visto simplemente como la punta de lanza del gran proceso de desintegración sociocultural que ha vivido históricamente la población andina, es el pastor quien encarna de manera más cruda su profundo poder destructor. Sólo un súper agente, avalado por la estructura cultural dominante, podría tener estas características. Especialmente cuando la mayoría de los actores pentecostales, aymaras y pentecostales aymaras, son concebidos como sujetos totalmente silentes dentro de la sociedad andina.

\section{Conclusión}

A la luz de la distinción analítica utilizada por Archer para evaluar la naturaleza ontológica y epistemológica de la explicación sobre el origen y desarrollo de los fenómenos socioculturales empleados por las distintas teorías socioculturales, podemos concluir lo siguiente respecto a los estudios sobre el pentecostalismo aymara en Chile.

En primer lugar, podemos apreciar que el uso de la distinción entre estructura y acción ha sido una herramienta conceptual ampliamente utilizada por estas investigaciones. Este uso ha seguido parcialmente el giro que han dado el resto de estudios sobre el pentecostalismo en Latinoamérica y también gran parte de la literatura de las ciencias sociales durante las últimas décadas. En concreto, se aprecia primeramente el predominio del uso de teorías y conceptos que tendían a explicar el fenómeno pentecostal basándose especialmente en la determinación macro de las estructuras sociales y culturales, siendo las acciones de los actores meras manifestaciones de este ordenamiento estructural. Posteriormente, se ha dado un giro que crecientemente ubica a los sujetos y sus acciones en un plano 
más activo en términos epistemológicos, otorgándoles incluso cierto nivel de construccionismo social. Sin embargo, los estudios sobre el pentecostalismo pentecostal aymara no han acabado de recorrer de manera íntegra este arco teórico que transita desde el predominio de teorías estructurales hasta el predominio de las teorías de la acción o la agencia.

En segundo lugar, y en estrecha relación con el punto anterior, podemos apreciar que si utilizamos la distinción teórica propuesta por Archer, podemos advertir que los estudios sobre el pentecostalismo aymara pueden ser clasificados en gran medida dentro de las teorías que explican la génesis y desarrollo de los fenómenos socioculturales a través de un conflacionismo descendente. Es decir, en la mayoría de los estudios analizados se concibe al movimiento pentecostal como un grupo socioreligioso que ha llegado a imponer una cultura foránea extraña a los aymaras, avalada por los ideales de la modernidad capitalista nacional y en un contexto de declive estructural de la población indígena. En esta línea argumentativa, la operación destructora del movimiento pentecostal, tanto a nivel de la ideología como de las prácticas sociales, evidencia finalmente la eficacia asociada a esta estructura sociocultural y la determinación que posee sobre el plano de las acciones.

En tercer lugar, debemos mencionar igualmente que los análisis han querido incorporar ciertamente la figura de la agencia de los actores. Por una parte, destacando el poder que poseen los actores pentecostales para interpretar y actuar en el mundo, lo que se evidencia claramente en su labor proselitista. Y por otra parte, admitiendo posteriormente también que la única forma de que se produjera un fenómeno tan importante como la conversión al pentecostalismo en la zona andina, fue gracias a la activa recepción que realizara la población aymara de esta nueva cosmovisión. Es decir, solo una vez que la doctrina pentecostal hizo sentido a los actores aymaras, estos pudieron finalmente adherirse al movimiento pentecostal, pasando a engrosar sus filas y abultando así las estadísticas locales y nacionales. En este punto, pareciera apreciarse cierto conflacionismo ascendente en estas explicaciones, sin embargo continúa predominando claramente el conflacionismo descendente como base de la explicación.

Quizás donde se manifiesta de manera más clara la figura de la agencia es en la construcción ideal que realizan todos los autores del pastor pentecostal, donde se le concibe como el gran gestor de la conversión al pentecostalismo y por ende del éxito del movimiento en la zona. Debido a una serie de características doctrinales que permiten identificar claramente a la figura del pastor, este sujeto aparece realmente como un súper actor. No sólo por el poder sociológico que en sí mismo le otorgan los autores, sino sobre todo por la desmedida eficacia que posee su accionar en comparación con la casi nula agencia atribuida a la inmensa mayoría de aymaras y pentecostales aymaras. Esta utilización conceptual presenta problemas a nivel empírico y epistemológico. Empírico porque es bastante cuestionable este desmedido poder atribuido al pastor en comparación con la baja agencia atribuida al resto de actores sociales que conforman esta realidad sociocultural; y epistemológico porque teóricamente no queda del todo claro dónde reside finalmente el motor explicativo de este fenómeno social. De manera que los rendimientos teóricos se ven limitados por estas preconcepciones teóricas y epistemológicas, pues finalmente caen de alguna u otra forma en un conflacionismo epistemológico.

\section{Agradecimiento}

Este artículo es parte del proyecto de investigación FONDECYT $\mathrm{N}^{\circ} 3120162$. Los autores agradecen a CONICYT Chile por el financiamiento. 


\section{Nota}

(1) El nivel de agencia observado por Guerrero se amplía y profundiza en las investigaciones de la década de 1990, cuando abandona completamente el funcionalismo y el estructural-funcionalismo, y comienza a apostar por una antropología de corte más interpretativo (e incluso posmoderna).

\section{Bibliografía}

Archer, M. 1997. El lugar de la cultura en la teoría social. Buenos Aires: Nueva Visión.

D’ Epinay, C. 1968. El refugio de las masas: estudio sociológico del protestantismo chileno. Santiago: Pacífico.

González, Y. 1980. La familia aymara: una estructura desintegrada y dominada. Cuaderno de Investigación Social 3: 34-38. http://crear.cl/wp-content/uploads//2014/02/3-Gonzalez-Yerny-CIS-3-ART3.pdf

González, S. 1990. El aymara de la provincia de Iquique-Chile y la educación nacional. Iquique: TER.

Gundermann, H. y González, H. 2009a. Sociedades indígenas y conocimiento antropológico. Aymaras y Atacameños de los siglos XIX y XX. Chungará 41: 113-164.

http://www.scielo.cl/pdf/chungara/v41n1/art08.pdf

Gundermann, H. y González, H. 2009b. Sujetos sociales andinos, antropología y antropólogos en Chile. ALPHA 29: 105-122. http://www.scielo.cl/pdf/alpha/n29/art08.pdf

Guerrero, B. 1980. La estructura ideológica del movimiento pentecostal. Iquique: CIRN.

Guerrero, B. 1984. Movimiento pentecostal, corrientes modernistas y sociedad aymara. Cuaderno de Investigación Social 8: 1-20. http://crear.cl/wp-content/uploads//2014/02/Cuaderno-InvestigacionSocial-8.pdf

Guerrero, B. 1992. Conversión y salud en el altiplano chileno. Revista de Ciencias Sociales 1: 30-36. http://www.redalyc.org/articulo.oa?id=70800103

Guerrero, B. 1993. Identidad aymara e identidad pentecostal: notas para la discusión. Revista de Ciencias Sociales 3: 23-36. http://www.revistacienciasociales.cl/archivos/revista3/pdf/rcs3 3.pdf

Guerrero, B. 1994. A Dios rogando...los pentecostales en la sociedad aymara del norte grande de Chile. Amsterdam: Vrije Universiteit Press.

Guerrero, B. 1998. La conversión al pentecostalismo. Una discusión teórica. Revista de Ciencias Sociales 8: 109-121. http://www.revistacienciasociales.cl/archivos/revista8/pdf/rcs8 8.pdf 
Kessler, J. 1967. A study of the older Protestant missions and churches in Perú and Chile. With special reference to the problems of division, nationalism and native ministry. Goes: Oosterbaan \& le Cointre N.V.

Mascareño, A. 2008. Acción, estructura y emergencia en la teoría sociológica. Revista de Sociología 22: 217-256. http://www.facso.uchile.cl/publicaciones/sociologia/articulos/22/2211-Mascareno.pdf

Pérez, E. 1975. La sociedad andina: una sociedad en desintegración. Cuaderno de Investigación Social 1: 57-74. http://crear.cl/wp-content/uploads//2014/02/5-Perez-CIS-1-ART-5.pdf

Tudela, P. 1993. Cambio religiosos y revitalización de la comunidad entre los aymaras de Arica 19601990. Nutram 33: 15-48.

Van Kessel, J. 2005. Holocausto al progreso. Los aymaras de Tarapacá. Ámsterdam: Cedla.

Van Kessel, J. y Guerrero, B. 1987. "Sanidad y Salvación" en el altiplano chileno: del yatiri al pastor. Cuaderno de Investigación Social 21: 1-44. http://crear.cl/wp-content/uploads//2014/02/CuadernoInvestigacion-Social-21.pdf

Willems, E. 1967. Followers of the new faith: Culture change and the rise of protestantism in Brazil and Chile. Tennessee: Vanderbilt University Press.

Recibido el 23 Ago 2014

Aceptado el 15 Oct 2014 